\title{
One-sided jet at milliarcsecond scales in LS I $+61^{\circ} 303$
}

\author{
M. Massi ${ }^{1}$, M. Ribó ${ }^{2}$, J. M. Paredes ${ }^{2}$, M. Peracaula ${ }^{2}$, and R. Estalella ${ }^{2}$ \\ 1 Max-Planck-Institut für Radioastronomie, Auf dem Hügel 69, 53121 Bonn, Germany \\ 2 Departament d'Astronomia i Meteorologia, Universitat de Barcelona, Av. Diagonal 647, 08028 Barcelona, Spain
}

\begin{abstract}
Received 5 March 2001 / Accepted 29 June 2001
Abstract. We present Very Long Baseline Interferometry (VLBI) observations of the high mass X-ray binary LS I $+61^{\circ} 303$, carried out with the European VLBI Network (EVN). Over the 11 hour observing run, performed $\sim 10$ days after a radio outburst, the radio source showed a constant flux density, which allowed sensitive imaging of the emission distribution. The structure in the map shows a clear extension to the southeast. Comparing our data with previous VLBI observations we interpret the extension as a collimated radio jet as found in several other X-ray binaries. Assuming that the structure is the result of an expansion that started at the onset of the outburst, we derive an apparent expansion velocity of $0.003 c$, which, in the context of Doppler boosting, corresponds to an intrinsic velocity of at least $0.4 c$ for an ejection close to the line of sight. From the apparent velocity in all available epochs we are able to establish variations in the ejection angle which imply a precessing accretion disk. Finally we point out that LS I $+61^{\circ} 303$, like SS 433 and Cygnus X-1, shows evidence for an emission region almost orthogonal to the relativistic jet.
\end{abstract}

Key words. stars: individual: LS I $+61^{\circ} 303$ - radio continuum: stars - X-rays: stars - stars: emission-line, Be stars: variables: general

\section{Introduction}

LS I $+61^{\circ} 303$ is an X-ray binary system associated with the galactic plane variable radio source GT $0236+610$ discovered by Gregory \& Taylor (1978). Optical observations (Hutchings \& Crampton 1981) show that the system is composed of a neutron star and an early type, rapidly rotating B0V star with a stable equatorial disk and mass loss. Spectral line observations of the radio source give a distance of $2.0 \pm 0.2 \mathrm{kpc}$ (Frail \& Hjellming 1991).

One of the most unusual aspects of its radio emission is the fact that it exhibits two periodicities: a 26.5 day periodic nonthermal outburst (Taylor \& Gregory 1982, 1984 ) and a 1584 day ( $~ 4$ years) modulation of the outburst peak flux (Gregory et al. 1999). The 26.5 day periodicity corresponds to the orbital period of the binary system (Hutchings \& Crampton 1981). This periodicity has also been detected in UBVRI photometric observations (Mendelson \& Mazeh 1989), in the infrared domain (Paredes et al. 1994), in soft X-rays (Paredes et al. 1997) and in the $\mathrm{H} \alpha$ emission line (Zamanov et al. 1999). The 4 year modulation has been observed as well in the $\mathrm{H} \alpha$ emission line (Zamanov et al. 1999).

Simultaneous X-ray and radio observations show that the X-ray outbursts occur at the periastron passage while,

Send offprint requests to: M. Massi,

e-mail: mmassi@mpifr-bonn.mpg.de on the contrary, the strongest radio outbursts are always delayed with respect to (Taylor et al. 1996; Gregory et al. 1999). Both the presence of two periodicities (at 26.5 days and $\sim 4$ years) and the delay between radio and X-ray outbursts are well explained in the framework of an accretion scenario of a precessing neutron star in a highly $(e>0.4)$ eccentric orbit (Gregory et al. 1989; Gregory et al. 1999). The accretion rate in an eccentric orbit within the equatorial wind of the Be star has two peaks: the highest peak corresponds to the periastron passage and the second, lower amplitude peak occurs when the relative velocity of the neutron star and the Be star wind is at a minimum. For supercritical accretion, matter is ejected outwards in two jets perpendicularly to the accretion disk plane. Near periastron, inverse Compton losses are severe (due to the proximity to the Be star): X-ray outbursts are expected but not radio ones. For the second accretion peak, the neutron star is much farther from the Be star and both inverse Compton losses and wind opacity are lower, the electrons can propagate out of the orbital plane and we observe the radio outburst. The precession of the disk gives rise to the $\sim 4$ year modulation (Gregory et al. 1989; Taylor et al. 1992; Massi et al. 1993; Martí \& Paredes 1995; Gregory et al. 1999). The presence of an accretion disk is also invoked by Mendelson \& Mazeh (1989) to explain details of the optical light curve. Liu et al. (2000) explain the variation of the $\mathrm{H} \alpha$ emission with orbital phase as varying 
irradiation of the Be star's circumstellar disk by the X-ray emission from the neutron star's accretion disk.

However, the luminosity of LS I $+61^{\circ} 303$ in the X-ray range $(1-40 \mathrm{keV})$, is only $L_{\mathrm{X}} \simeq 10^{35} \mathrm{erg} \mathrm{s}^{-1}$ (Maraschi \& Treves 1981), which is three orders of magnitude lower than the Eddington limit. Moreover, LS I $+61^{\circ} 303$ is the most promising candidate for the optical counterpart of the $\gamma$-ray source 3EG J0241+6103 (Hartman et al. 1999), with a luminosity $L_{\gamma} \simeq 10^{37} \mathrm{erg} \mathrm{s}^{-1}$. The fact that LS I $+61^{\circ} 303$ has the bulk of its energy shifted from X-ray to $\gamma$-ray wavelengths is not understood in the context of the supercritical accretion model, showing the necessity for alternative models. These models suggest that the $26.5 \mathrm{~d}$ outburst events are produced by energetic electrons accelerated in the shock boundary between the relativistic wind of a young non-accreting pulsar and the wind of the Be star. In this case the 4 year modulation would be explained due to cyclic variations of the Be star envelope (Maraschi \& Treves 1981; Tavani 1994; Tavani et al. 1998; Goldoni \& Mereghetti 1995; Zamanov 1995; Taylor et al. 1996).

The recent discovery of the microquasar LS 5039 (Paredes et al. 2000) brings new credibility to the accretion model. LS 5039 is also subluminous in the X-ray range (even more than LS I $+61^{\circ} 303$ ) and also shows the same puzzling behavior, having $L_{\gamma}>L_{\mathrm{X}}$. Therefore, LS 5039 and LS I +61 303 could be the first two examples of a new class of X-ray binaries with powerful $\gamma$-ray emission. In the case of LS 5039, an ejection process fed by an accretion disk is clearly proved by a map obtained with the Very Long Baseline Array (VLBA), which shows bipolar jets emerging from a central core. Its high $L_{\gamma}$ is tentatively explained by inverse Compton scattering. In LS I $+61^{\circ} 303$, although several VLBI observations show a complex source extending over a few milliarcseconds (Massi et al. 1993; Peracaula et al. 1998; Paredes et al. 1998; Taylor et al. 2000), such a clear jet structure has never been observed.

In this paper we report on EVN observations probing structures on scales of tens of milliarcseconds, larger than probed by previous observations. We clearly detect on this scale an elongation of the emission (Sect. 2), which we interpret in Sect. 3 as a one-sided jet. We determine its apparent expansion velocity and derive the intrinsic velocity considering Doppler boosting. Our conclusions are given in Sect. 4.

\section{Observations and results}

Our observations of LS I $+61^{\circ} 303$ were carried out during 11 hours on 1994 June 7 , at $5 \mathrm{GHz}$, using four antennas of the EVN, namely the Effelsberg $100 \mathrm{~m}$, Medicina $32 \mathrm{~m}$, Noto $32 \mathrm{~m}$ and Onsala $25 \mathrm{~m}$ telescopes. The data were recorded, in left hand circular polarization, using the Mark III mode A recording system, corresponding to a total bandwidth of $56 \mathrm{MHz}$. The correlation of the data was done on the Mark III correlator operated by the MaxPlanck-Institut für Radioastronomie in Bonn.

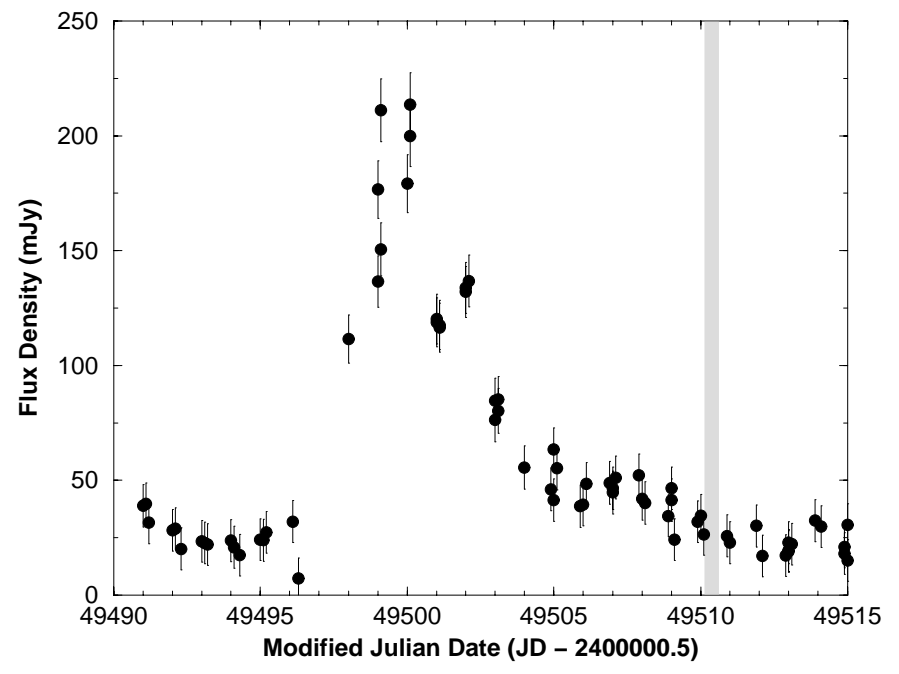

Fig. 1. Radio light curve of LS I $+61^{\circ} 303$ obtained with the GBI at $8.4 \mathrm{GHz}$ (Strickman et al. 1998). The shaded area indicates the time interval during which our VLBI data were taken.

Our EVN observations were made within a time interval during which a multiwavelength monitoring campaign was conducted that included simultaneous radio, optical, infrared, and hard X-ray $/ \gamma$-ray observations and covered almost three orbital cycles of LS I $+61^{\circ} 303$ (Strickman et al. 1998). As can be seen from the radio light curve taken with the Green Bank Interferometer ${ }^{1}$ (GBI) as part of that campaign (Fig. 1), our observations took place about 10 days after the radio outburst maximum (i.e. almost 13 days after the onset of the outburst). Total flux density observations of LS I $+61^{\circ} 303$, carried out by the $100 \mathrm{~m}$ telescope of Effelsberg at the beginning of each VLBI scan, are shown in Fig. 2. The variations of left and right hand circular polarization flux are within their error bars. This lack of significant circular polarization agrees with previous results of Peracaula et al. (1997). The total flux density is nearly constant and with an average value of $34 \pm 5 \mathrm{mJy}$.

In our EVN observations, LS I +61 303 was clearly detected on all baselines. The data were reduced using standard procedures within the AIPS and Difmap software packages. The EVN uniform weighted map is shown at the top of Fig. 3.

It exhibits an extended structure elongated to the southeast. The elongation is not artificially created by the beam, in fact the elongation is at $\mathrm{PA} \simeq 120^{\circ}$ while the beam, of size 5.9 mas $\times 3.8$ mas, has $\mathrm{PA}=74.2^{\circ}$. The flux density recovered in the cleaning process amounts to $\sim 35 \mathrm{mJy}$.

In Table 1 we show the parameters obtained after fitting several models to the $(u, v)$ data. The best fit using a

\footnotetext{
1 The Green Bank Interferometer is a facility of the USA National Science Foundation operated by the NRAO in support of NASA High Energy Astrophysics programs.
} 
Table 1. Parameters of different models fitted to the data.

\begin{tabular}{lllllllll}
\hline \hline Model & $\chi_{\mathrm{r}}^{2}$ & Components & $\begin{array}{l}\text { Flux Density } \\
(\mathrm{mJy})\end{array}$ & $\begin{array}{l}r \\
(\mathrm{mas})\end{array}$ & $\begin{array}{l}\theta \\
\left({ }^{\circ}\right)\end{array}$ & $\begin{array}{l}\text { Maj. axis } \\
(\mathrm{mas})\end{array}$ & $\begin{array}{l}\text { Min. axis } \\
(\mathrm{mas})\end{array}$ & $\begin{array}{l}\text { PA } \\
\left(^{\circ}\right)\end{array}$ \\
\hline \hline 1 & 0.798 & Elliptical Gaussian & 33.0 & 0.04 & 3 & 3.7 & 2.5 & 123 \\
\hline 2 & 0.786 & Elliptical Gaussian & 21.6 & 0.5 & 135 & 5.5 & 3.6 & 123 \\
& & Point source & 13.1 & 0.5 & -43 & & & \\
\hline \hline
\end{tabular}

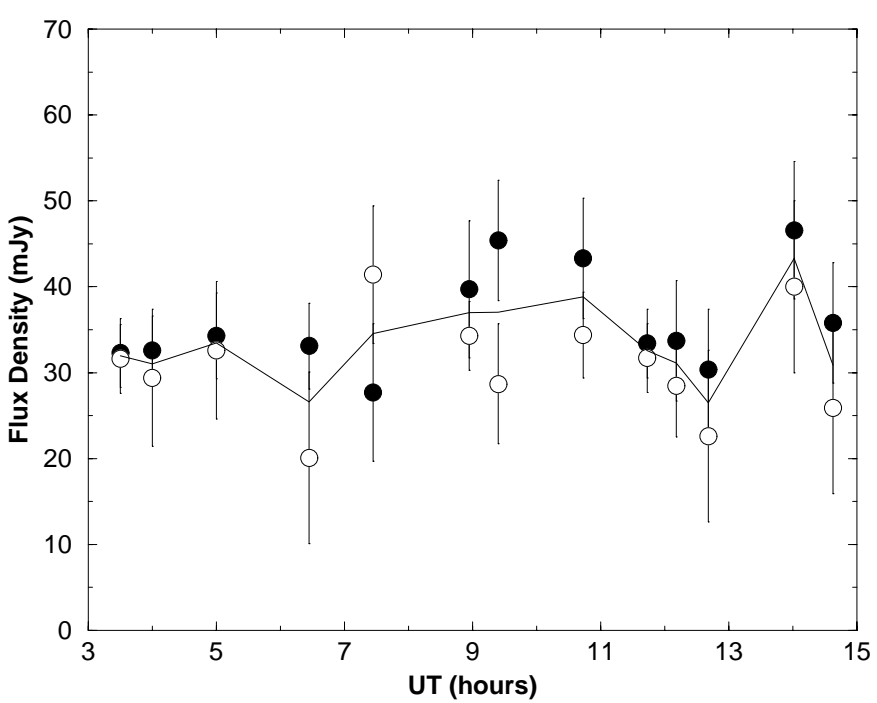

Fig. 2. Effelsberg flux density measurements of LS I $+61^{\circ} 303$ at $5 \mathrm{GHz}$ obtained during our VLBI observations. LCP and RCP flux density measurements are represented by filled and open circles respectively. The solid line corresponds to the total flux density.

single component is obtained with an elliptical Gaussian (Model 1 in Table 1). A circular Gaussian or a point source gives higher values of $\chi_{r}^{2}$. The elliptical Gaussian has a PA consistent with the observable elongation in the map. However, there is still residual emission not accounted for by Model 1, in the direction of the elongation.

In previous VLBI observations (Paredes et al. 1998) the best fits to the $(u, v)$ data were consistent with an unresolved core plus a Gaussian halo. Hence, we have added a point source to Model 1, and we have obtained a slightly improved fit to the data (Model 2 in Table 1). It is important to note that, in this model, the elliptical Gaussian conserves its PA and moves towards the direction of the elongation, while the point source moves in the opposite direction. We have tried several different initial conditions and always the fit converged to the same solution. Finally, for a distance of $2.0 \mathrm{kpc}$ (Frail \& Hjellming 1991), the brightness temperature of the components of the models listed in Table 1 are in the range of $T_{\mathrm{B}}=10^{7}-10^{8} \mathrm{~K}$, which are characteristic of non-thermal emission.

\section{Discussion}

\subsection{Morphology}

In view of the models that can fit the data, the source in Fig. 3 can be explained as a central core (point source of

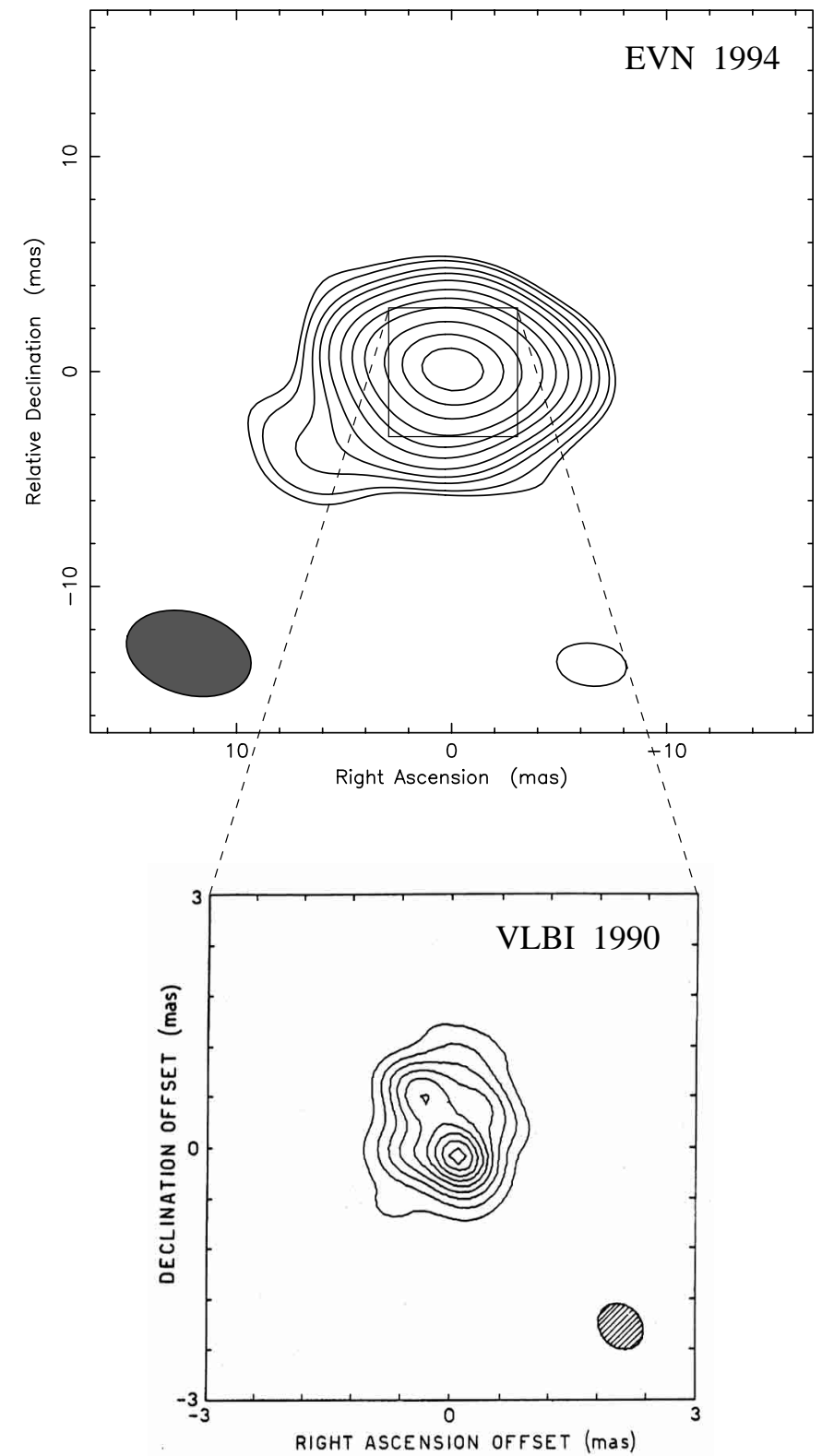

Fig. 3. Top: EVN uniform weighted map of LS I $+61^{\circ} 303$ at $5 \mathrm{GHz}$ frequency obtained on 1994 June 7 (this paper). The contours are at $-3,3,4,6,8,11,16,22,30,45,60$ and 75 times the rms noise of $0.28 \mathrm{mJy}_{\text {beam }}^{-1}$. The filled ellipse in the bottom-left corner represents the FWHM of the synthesized beam, which is 5.9 mas $\times 3.8$ mas at a PA of $74.2^{\circ}$. Bottom: VLBI map (at $5 \mathrm{GHz}$ ) by Massi et al. (1993), obtained on 1990 June 6 , with a resolution of 0.6 mas $\times 0.5$ mas. 
Model 2 in Table 1) and a one-sided jet (elliptical Gaussian in the same model). If we assume that the Gaussian component is the result of an expanding source that originated at the onset of the major outburst, that is about 13 days before our observations, and that the expansion velocity of the jet is constant, the derived velocity, taking into account the size of the Gaussian and the offset to the point source, is $\sim 0.3$ mas d$^{-1}$. This corresponds, at a $2.0 \mathrm{kpc}$ distance, to a projected expansion velocity of $\sim 0.003 \mathrm{c}$.

The most interesting aspect of the EVN map is that, for the first time, we detected asymmetric emission in the southeast direction. The morphology is quite similar to that observed by Stirling et al. (2000, Fig. 4) in Cygnus X-1 at 15 GHz: a central source with a small but clear elongation. Is this morphological analogy enough to interpret the structure in the EVN map as a one-sided jet? In the case of Cygnus X-1 the interpretation of the small jet-like elongation has proved to be correct: a later observation of this source at $8 \mathrm{GHz}$ shows the elongation developed into an extended jet.

LS I $+61^{\circ} 303$ has been observed several times with mas resolution. In Table 2 we report previous VLBI observations focusing on two special items: the position angle (PA) of any extended feature present and its expansion velocity. As can be seen, an extended feature with PA from $120^{\circ}$ to $160^{\circ}$ seems to be present in other maps. While the identification is perhaps ambiguous in Paredes et al. (1998) because the extension is coincident with the direction of the major axis of the beam, the observations by Peracaula et al. (1998), report on one extended feature with comparable PA with that of the feature in our map. The reason why in the past this extended structure was never clearly associated with a jet is because an internal structure at another $\mathrm{PA}\left(\simeq 30^{\circ}\right)$ makes the morphology confusing. To better understand this point we show at the bottom of Fig. 3 the map by Massi et al. (1993). The VLBI map resolves two components $\left(\right.$ at $\left.\mathrm{PA} \simeq 30^{\circ}\right)$ inside the extended structure $\left(\right.$ at $\mathrm{PA} \simeq 135^{\circ}$ ). The extended structure is asymmetric as well as that in our map, but towards the northwest direction. This is probably a projection effect corresponding to a different ejection angle due to precession (Sect. 3.3). In Paredes et al. (1998) the double internal source is not resolved, but the authors propose the presence of an unresolved component stable and not participating in the flaring process, inside an extended structure at $\mathrm{PA} \simeq 120^{\circ}$. These two components are again resolved in the recent VLBI observation using HALCA (Taylor et al. 2000). While in the past this double internal source was supposed to be the jet, in light of the EVN map we propose that:

1. the double source at PA of $\simeq 30^{\circ}$ is not the jet. Instead, it is almost orthogonal to the jet. It has a size of $2 \mathrm{AU}$ and therefore is comparable with the orbital size (1.4 AU);

2. the jet is identified with the structure with PA in the range $120^{\circ}-160^{\circ}$ seen in at least three VLBI observations, and best defined in our EVN map where it has a size of $\sim 8 \mathrm{AU}$.

An emitting region perpendicular to the jet has been observed indeed also in SS 433 by Paragi et al. (1999) and perhaps in Cygnus X-1 by Stirling et al. (2000) and preliminary interpreted as shocked gas in the orbital/accretion plane. If this interpretation holds true also for LS I $+61^{\circ} 303$ the strong wind opacity close to the periastron, discussed in the introduction, certainly severely affects the morphology of such emission. For the jet, however, wind opacity effects are negligible. The jet is generated by outbursts occurring at quite a displaced orbital phase with respect to the periastron. Moreover, the size of the jet is much greater than the orbital size.

\subsection{One-sided jet and Doppler boosting}

It is well known that while some extragalactic radio sources have two jets, for some others only one jet is observed. The unification model for AGN (see a review in Urry \& Padovani 1995) assumes that all of them represent the same class of objects (all having two jets) and their different appearance depends on different observing angles. Let us assume a symmetric ejection of two jets at velocity $\beta$ (i.e. expressed as fraction of $c$ ). The two jets, approaching and receding, move at an apparent velocity $\beta_{\mathrm{a}, \mathrm{r}}$ related to the intrinsic $\beta$ by (Rees 1966; Mirabel \& Rodríguez 1994)

$\beta_{\mathrm{a}, \mathrm{r}}=\frac{\beta \sin \theta}{1 \mp \beta \cos \theta}$,

where $\theta$ is the angle between the direction of motion of the ejecta and the line of sight.

Following the method of Mirabel \& Rodríguez (1994) one can determine the quantity $\beta \cos \theta$ by means of the ratio of flux densities from the approaching and receding jets,

$\frac{S_{\mathrm{a}}}{S_{\mathrm{r}}}=\left(\frac{1+\beta \cos \theta}{1-\beta \cos \theta}\right)^{k-\alpha}$

where $\alpha$ is the spectral index of the emission $\left(S \propto \nu^{\alpha}\right)$ and $k$ is 2 for a continuous jet and 3 for discrete condensations.

In our case we deal with one jet only. However, we can determine the lower limit

$\beta \cos \theta>\frac{\left(S_{\mathrm{a}}^{\text {peak }} / 3 \sigma\right)^{1 /(k-\alpha)}-1}{\left(S_{\mathrm{a}}^{\text {peak }} / 3 \sigma\right)^{1 /(k-\alpha)}+1}$,

using the noise level $(\sigma)$ of the map and the peak value of the approaching component $\left(S_{\mathrm{a}}^{\text {peak }}\right)$. The spectral index a few days after the outburst is $\alpha=-0.5$ (Strickman et al. 1998). To be consistent with the lowest limit we select $k=3$.

For our EVN map, with $\sigma=0.28 \mathrm{mJy}^{\text {beam }}{ }^{-1}$ and $S_{\mathrm{a}}^{\text {peak }}=21.6 \mathrm{mJy} \mathrm{beam}^{-1}$, we have $\beta \cos \theta>0.43$.

Using Eq. (1) we obtain

$\beta=\sqrt{(\beta \cos \theta)^{2}+\beta_{\mathrm{a}}{ }^{2}(1-\beta \cos \theta)^{2}}$, 
Table 2. Summary of published VLBI observations of LS I $+61^{\circ} 303$ at $5 \mathrm{GHz}$. Phase is computed according to $P=26.4917 \mathrm{~d}$ and $T_{0}=$ JD 2443366.775 .

\begin{tabular}{|c|c|c|c|c|c|c|c|}
\hline Epoch & Array & Phase & State & $\begin{array}{l}\text { Flux Density } \\
(\mathrm{mJy})\end{array}$ & $\begin{array}{l}\text { Size } \\
\text { (mas) }\end{array}$ & $\begin{array}{l}\text { PA } \\
\left({ }^{\circ}\right)\end{array}$ & $\begin{array}{l}\text { Expansion } \\
\text { Velocity/c }\end{array}$ \\
\hline 1987 Sep. $25^{a}$ & \multirow[b]{2}{*}{$\mathrm{EVN}$} & 0.59 & quiescent & $40-54$ & $3.2 \pm 0.9$ & & \multirow[b]{2}{*}{$\leq 0.002$} \\
\hline 1987 Oct. $1^{a}$ & & 0.81 & burst, decaying & $260-200$ & $1.6 \pm 1.2$ & & \\
\hline 1990 Jun. $6^{b}$ & $\mathrm{EVN}+\mathrm{VLA}$ & 0.74 & burst, decaying & $244-205$ & $\sim 2$ & $\sim 135, \sim 30$ & $\sim 0.002^{1}$ \\
\hline 1992 Jun. $8^{c}$ & Global VLBI & 0.42 & quiescent, minioutburst & 35 & $0.5-2$ & $\sim 160$ & 0.06 \\
\hline 1993 Sep. $9^{d}$ & \multirow[b]{2}{*}{ Global VLBI } & 0.69 & burst, variable & $76-131$ & $2-3$ & & \multirow[b]{2}{*}{0.007} \\
\hline 1993 Sep. $13^{d}$ & & 0.84 & quiescent & 60 & $\sim 7$ & $\sim 120$ & \\
\hline 1994 Jun. $7^{e}$ & $\mathrm{EVN}$ & 0.92 & quiescent & 34 & $\sim 6$ & $\sim 120$ & 0.003 \\
\hline 1999 Sep. $16-17^{f}$ & $\begin{array}{l}\text { HALCA + } \\
\text { Global VLBI }\end{array}$ & 0.69 & burst, variable & 140 & $\sim 4$ & & $\sim 0.002^{2}$ \\
\hline
\end{tabular}

${ }^{a}$ Taylor et al. (1992); ${ }^{b}$ Massi et al. (1993); ${ }^{c}$ Peracaula et al. (1998); ${ }^{d}$ Paredes et al. (1998); ${ }^{e}$ This paper; ${ }^{f}$ Taylor et al. (2000).

${ }^{1}$ Recalculated according to new outburst peak ephemerides (Gregory et al. 1999), for the extended structure at PA $=135^{\circ}$.

2 There is not enough resolution at $\mathrm{PA} \sim 140$ to know if there was expansion in that direction.

which for $\beta_{\mathrm{a}}=0.003$ (Sect. 3.1) and $\beta \cos \theta>0.43$ gives an intrinsic velocity of $\beta>0.43$ and an ejection angle $\theta \simeq 0^{\circ}$. If we consider a typical size of $\sim 10^{-2} \mathrm{pc}$ for the jets in other known microquasars, the $\sim 8 \mathrm{AU}$ size of the LS I $+61^{\circ} 303$ jet would result in an angle of $\theta \simeq 0.2$, compatible with our estimation.

As reviewed by Mirabel \& Rodríguez (1999), the expansion velocities for microquasars range from $\sim 0.1 c$ to $\sim 0.9 \quad c \quad$ SS 433, Cygnus X-3, GRS 1915+105, GRO J1655-40). The above determined lower limit of $0.4 c$ for $\mathrm{LS} \mathrm{I}+61^{\circ} 303$ is therefore well within that range. Finally, we note that the combination of the values estimated for $\theta$ and $\beta$ gives a Lorentz factor $\gamma=1.1$, and a Doppler factor $\delta_{\mathrm{a}, \mathrm{r}}=\frac{1}{\gamma(1 \mp \beta \cos \theta)}, \delta_{\mathrm{a}}=1.59$ for the approaching jet and $\delta_{\mathrm{r}}=0.63$ for the receding one.

\subsection{Precession of the accretion disk}

The measured expansion velocities shown in Table 2 span a range of $0.002-0.007 c$ and reach a value of $0.06 c$ at epoch 1992 June 8. On the basis of our discussion in Sects. 3.1 and 3.2, we interpret the observed expansion velocities as apparent transverse velocities, $\beta_{\mathrm{a}}$, defined by Eq. (1). A possible explanation for the large range of observed velocities is a variable intrinsic velocity $\beta$ and a constant $\theta$. However, this is not supported by the observations available up to now; moreover, for example, SS 433 has shown a quite constant velocity of $\beta=0.26$ for years. The alternative explanation is precession of the jet, with the angle $\theta$ between the direction of the jet and the line of sight being a function of time. Evidence for precession has been found at least for SS 433 and Cygnus X-1 (Brocksopp et al. 1999). Moreover, for LS I $+61^{\circ} 303$, precession of the jet has already been suggested to explain the $4 \mathrm{yr}$ modulation of the peak of the radio outbursts (Gregory et al. 1989).

If the latter assumption is correct, we would expect an anticorrelation between the flux density of the radio outburst peak, and $\theta$ or $\beta_{\mathrm{a}}$. In other words, when the jet is pointing directly towards us ( $\theta$ small), $\beta_{\mathrm{a}}$ is also small, and the flux density of the outburst peak is the highest possible due to the Doppler boosting effect. On the contrary, when the jet is not pointing directly towards us, $\theta$ and $\beta_{\mathrm{a}}$ increase, and the flux density decreases. In Fig. 4 we show the flux density of the radio outburst peak versus $\log \beta_{\mathrm{a}}$, for the VLBI observations listed in Table 2, taking $\beta_{\mathrm{a}}$ as the observed expansion velocity. We can see from Fig. 4 that the available data are consistent with the anticorrelation of the flux density of the radio outburst peak and $\beta_{\text {a }}$ predicted by our model, and thus with the precession of the jet of LS I $+61^{\circ} 303$.

Assuming that the the intrinsic velocity of the jet remains constant $(\beta \simeq 0.4)$ for all the observations listed in Table 2, we can derive that the maximum value of the ejection angle $\theta$, corresponding to the maximum value of $\beta_{\mathrm{a}}=0.06$, is $\theta \simeq 4.5$. This maximum angle is consistent with a moderate precession of the jet, and could be the result of the precession of the accretion disk.

The range of values obtained for $\theta$ imply that the orbital plane of the system would be close to the plane of the sky, i.e., the inclination of the orbit, $i$, should be small. The other available information about $i$ comes from 


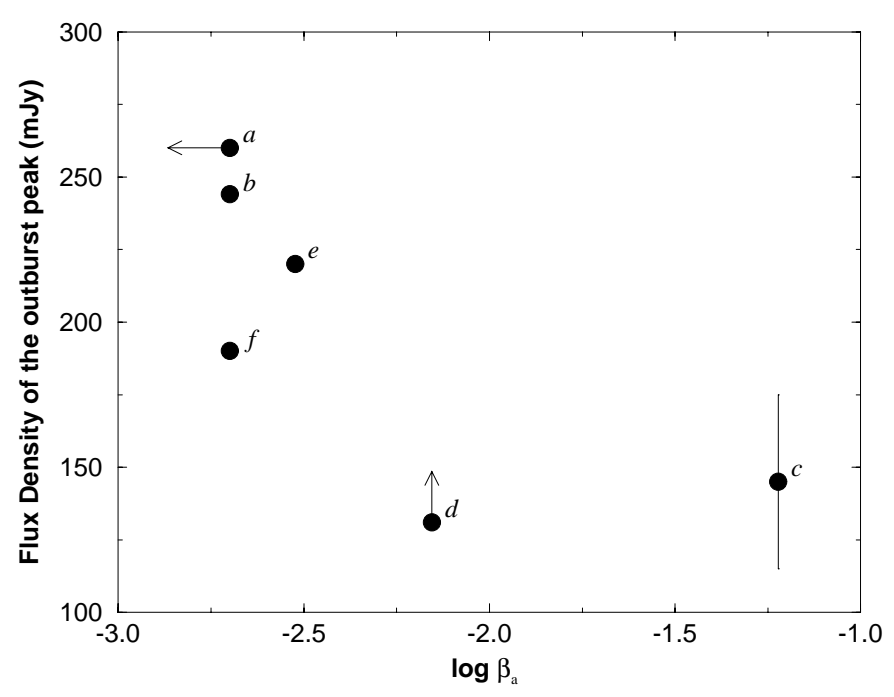

Fig. 4. Flux density of the radio outburst peak versus $\log \beta_{\mathrm{a}}$ (see text). Each data point in this diagram is the result of the corresponding VLBI observation identified with letters $a$ to $f$ (see Table 2). For observations $a, b, e$ and $f$ the flux density of the radio outburst peak was known from the same VLBI observations or from the GBI monitoring program. Point $d$ is a lower limit of the outburst peak, while $c$ is taken from the average outburst peak flux modulation of Gregory et al. (1999).

spectroscopic optical and UV observations carried out by Hutchings \& Crampton (1981). Although their observations reveal shell absorption, this fact alone does not give information on the angle of inclination, because the shell can cover the whole star and not be just an equatorial bulge (Kogure 1969; Geuverink 1970). We note that the observations by Waters et al. (1988) establish that, together with a dense and slow disk-like wind around the equator, there exists a high velocity, low density wind at higher latitudes up to the polar regions. On the other hand, Hutchings \& Crampton (1981) obtain $v \sin i=$ $360 \pm 25 \mathrm{~km} \mathrm{~s}^{-1}$, where $v$ is the equatorial rotational velocity of the Be star. As the maximum rotational velocity of a Be star (Hutchings et al. 1979) is $v=630 \mathrm{~km} \mathrm{~s}^{-1}$, this would result in a value of $i \sim 35^{\circ}$. However, Hutchings \& Crampton (1981) comment that the velocity data are "extensive and unwieldy" and indeed the value of $v \sin i$ given above seems large if compared with the statistical study of Be stars by Slettebak (1982). New observations would be very useful to clarify this issue.

\section{Conclusions}

Our EVN observations at $5 \mathrm{GHz}$ revealed that LS I $+61^{\circ} 303$ has a jet-like elongation to the southeast on scales of tens of milliarcseconds. This is interpreted as an approaching, Doppler boosted, jet of a symmetric pair. The angle between the jet and the line of sight at the epoch of our observation was close to zero. However, data at other epochs suggest the existence of precession of the jet, and the accretion disk, of at least $5^{\circ}$. This precession would change the angle of the ejecta and consequently would vary both the apparent expansion velocity and the intensity of the peak of the radio outbursts. New VLBI observations at several epochs are necessary to correlate morphology, position angle, and expansion velocity, with the $4 \mathrm{yr}$ modulation of the peak of the radio outbursts (Gregory et al. 1999) and of the $\mathrm{H} \alpha$ emission line (Zamanov et al. 1999).

We derived a lower limit of $0.4 c$ for the intrinsic velocity of the radio jet. This value is well within the range $0.1 c$ to $0.9 c$ found for microquasars like SS 433, Cygnus X-3, GRS 1915+105 and GRO J1655-40 (Mirabel \& Rodríguez 1999).

Finally, in some microquasars, emission almost orthogonal to the jet, i.e. along the orbital plane, exists. This emission has clearly been observed in SS 433 (Paragi et al. 1999 ) and it is barely visible in Cygnus X-1 (Stirling et al. 2000). As we showed in this paper, LS I +61 303 could be a third case of this kind of source.

Acknowledgements. We thank Karl Menten, Andrew Lobanov, Alok Patnaik, Eduardo Ros and Giovanna Pugliese for useful discussions and comments. We acknowledge detailed and very useful comments from L. Lara, the referee of this paper. During this work, M.R. has been supported by two fellowships from CIRIT (Generalitat de Catalunya, Ref. 1998 BEAI 200293 and 1999 FI 00199). J. M. P. and M. P. acknowledge support from DGICYT grant PB97-0903 (Spain).

\section{References}

Brocksopp, C., Fender, R. P., Larionov, V., et al. 1999, MNRAS, 309, 1063

Frail, D. A., \& Hjellming, R. M. 1991, AJ, 101, 2126

Geuverink, H. G. 1970, A\&A, 5, 341

Goldoni, P., \& Mereghetti, S. 1995, A\&A, 299, 751

Gregory, P. C., \& Taylor, A. R. 1978, Nature, 272, 704

Gregory, P. C., Huang-Jian, X., Backhouse, C. J., \& Reid, A. 1989, ApJ, 339, 1054

Gregory, P. C., Peracaula, M., \& Taylor, A. R. 1999, ApJ, 520, 376

Hartman, R. C., Bertsch, D. L., Bloom, S. D., et al. 1999, ApJS, 123, 79

Hutchings, J. B., Nemec, J. M., \& Cassidy, J. 1979, PASP, 91, 313

Hutchings, J. B., \& Crampton, D. 1981, PASP, 93, 486

Kogure, T. 1969, PASJ, 21, 71

Liu, Q. Z., Hang, H. R., Wu, G. J., Chang, J., \& Zhu, Z. X. 2000, A\&A, 359, 646

Maraschi, L., \& Treves, A. 1981, MNRAS, 194, 18

Martí, J., \& Paredes, J. M. 1995, A\&A, 298, 151

Massi, M., Paredes, J. M., Estalella, R., \& Felli, M. 1993, A\&A, 269, 249

Mendelson, H., \& Mazeh, T. 1989, MNRAS, 239, 733

Mirabel, I. F., \& Rodríguez, L. F. 1994, Nature, 371, 46

Mirabel, I. F., \& Rodríguez, L. F. 1999, ARA\&A, 37, 409

Paragi, Z., Vermeulen, R. C., Fejes, I., et al. 1999, A\&A, 348, 910

Paredes, J. M., Marziani, P., Martí, J., et al. 1994, A\&A, 288, 519 
Paredes, J. M., Martí, J., Peracaula, M., \& Ribó, M. 1997, Tavani, M., Kniffen, D., Mattox, J. R., Paredes, J. M., \& A\&A, 320, L25 Foster, R. S. 1998, ApJ, 497, L81

Paredes, J. M., Massi, M., Estalella, R., \& Peracaula, M. 1998, A\&A, 335, 539

Taylor, A. R., \& Gregory, P. C. 1982, ApJ, 255, 210

Taylor, A. R., \& Gregory, P. C. 1984, ApJ, 283, 273

Paredes, J. M., Martí, J., Ribó, M., \& Massi, M. 2000, Science, 288,2340

Peracaula, M., Martí, J., \& Paredes, J. M. 1997, A\&A, 328, 283

Taylor, A. R., Kenny, H. T., Spencer, R. E., \& Tzioumis, A. 1992, ApJ, 395, 268

Taylor, A. R., Young, G., Peracaula, M., Kenny, H. T., \& Gregory, P. C. 1996, A\&A, 305, 817

Peracaula, M., Gabuzda, D. C., \& Taylor, A. R. 1998, A\&A, 330,612

Rees, M. J. 1966, Nature, 211, 468

Slettebak, A. 1982, ApJS, 50, 55

Stirling, A. M., Spencer, R. E., De la Force, C. J., et al. 2000, MNRAS, in press

Strickman, M. S., Tavani, M., Coe, M. J., et al. 1998, ApJ, 497, 419

Taylor, A. R., Dougherty, S. M., Scott, W. K., Peracaula, M., \& Paredes, J. M. 2000, proc. of Astrophysical Phenomena Revealed by Space VLBI, ed. H. Hirabayashi, P. G. Edwards, \& D. W. Murphy, 223

Urry, C. M., \& Padovani, P. 1995, PASP, 107, 803

Waters, L. B. F. M., van den Heuvel, E. P., Taylor, A. R., Habets, G. M. H. J., \& Persi, P. 1988, A\&A, 198, 200

Zamanov, R. K. 1995, MNRAS, 272, 308

Tavani, M. 1994, in The gamma-ray Sky with GRO and SIGMA (Dordrecht, Kluwer), 181

Zamanov, R. K., Martí, J., Paredes, J. M., et al. 1999, A\&A, 351,543 